\title{
ANTI-TUBERCULOSIS DRUG RESISTANCE PATTERN AMONG DIFFERENT CATEGORY OF TUBERCULOSIS PATIENTS
}

\author{
MOSTAFIZUR RAHMAN ${ }^{1}$, SM MOSTAFA KAMAL ${ }^{2}$, FAZLE RABBI MOHAMMED ${ }^{3}$, MD. BILLAL ALAM ${ }^{4}$, \\ HAM NAZMUL AHASAN ${ }^{5}$
}

\begin{abstract}
:
Background: Anti-Tuberculosis (TB) drug resistance is emerging as a new global health problem. No national data on drug resistance is available in Bangladesh. The absolute number of multidrug resistant TB (MDR-TB) is expected to be high considering high TB burden. This study was aimed to determine the resistance pattern of mycobacterium tuberculosis (MTB) isolates among different category of patient.

Method: A total 1123 randomly selected patients having clinical and or radiological features of tuberculosis attending out patients department of NIDCH were enrolled in this study during January to December, 2008. Sputum were collected and processed for culture by digestion, decontamination and concentration following modified Petroff's method and were inoculated on to two slopes of Lowenstein- Jensen (L-J) media for six weeks. The identity of the isolates was made by growth rate, colony morphology, P-nitrobenzoic acid (PNB) susceptibility, catalase and nitrate reduction tests. Ultimately drug susceptibility testing (DST) were performed.

Result: Drug susceptibility testing for Isoniazid (INH), Rifampicin (RIF), Ethambutol (EMB) and Streptomycin (SM) was done among 363 cases. Resistance rates for INH, RIF, EMB and SM were $76.03 \%, 71.63 \%, 27.55 \%$ and $55.65 \%$ respectively. According to DST report, total 221 cases were detected as multi drug resistant TB (MDR-TB). Among them, $87 \%$ cases were MDR-TB in category II (CAT-II) failure and $13 \%$ were MDR-TB in category I (CAT-I) failure and it was $0 \%$ in new cases.

Conclusion: Pattern of anti TB drug resistance was identified in this study. More surveillance and immediate therapeutic interventions should be performed in order to combat the threat of MDR-TB to the general population.
\end{abstract}

Keywords: Tuberculosis, Multi drug resistance, Drug susceptibility testing.

\section{Introduction:}

Tuberculosis (TB) remains a major public health issue from a single infectious agent in Bangladesh. According to current estimates, one person gets ill every two minutes and one person dies every ten minutes. Over 300000 new TB cases and 70,000 deaths are estimated per year in Bangladesh and the country ranks fifth on the global list of high TB burden countries. ${ }^{1,2}$ Multidrug-resistant TB (MDR-TB) includes drug resistance to at least Isoniazid (INH) and Rifampicin (RIF). No national study has been conducted to evaluate the current status of MDR-TB in Bangladesh. Some smaller studies in Dhaka reveal different levels of MDR-TB. This shows 1.4\% MDRTB in new cases and $20 \%$ in re treatment cases. ${ }^{1}$ Another study conducted outside Dhaka shows up to $90 \%$ MDR-TB in previously retreated failure cases. ${ }^{1}$ WHO estimates there are nearly half a million new cases of MDR-TB, about $5 \%$ of the total nine million new TB cases, worldwide each year. ${ }^{3}$ In this study, we aimed to determine the resistance pattern of mycobacterium tuberculosis (MTB) isolates among different category of patient.

1. Director \& Professor, National Institute of Diseases of Chest and Hospital (NIDCH), Dhaka

2. Assistant Professor, Dept. of Pathology, NIDCH, Dhaka.

3. Post-graduate trainee, Department of Medicine, Dhaka Medical College Hospital, Dhaka.

4. Associate Professor, Department of Medicine, Dhaka Medical College, Dhaka

5. Professor and Head, Department of Medicine, Dhaka Medical College, Dhaka

Correspondence : Dr. Mostafizur Rahman, Director \& Professor, National Institute of Diseases of Chest and Hospital (NIDCH), Dhaka 


\section{Materials and Method:}

A total 1123 randomly selected patients having clinical and or radiological features of tuberculosis attending out patients department of NIDCH were enrolled in this study during January to December, 2008. One spot sputum specimen (about $5 \mathrm{ml}$ ) and another morning specimen were collected under the guidance of a doctor. Both the specimen was sent to laboratory as soon as possible. Two direct smears were prepared from each of the two specimens for staining by ZiehlNelsen (Z-N) and Flurochrome (FM) techniques and were examined under microscope following standard operating procedure (SOP). Each specimen was processed for culture by digestion, decontamination and concentration following modified Petroff's method and was inoculated on to two slopes of LowensteinJensen (L-J) media for six weeks. The identities of the isolates were made by growth rate, colony morphology, P-nitrobenzoic acid (PNB) susceptibility, catalase and nitrate reduction tests. Drug susceptibility testing (DST) was performed for Isoniazid, Rifampicin, Ethambutol (EMB) and Streptomycin (SM) after isolation by using the resistance proportion method. The study protocol was approved by the institutional ethics committee. Informed written consent was taken from each patient or his / her guardian. Analysis of data was performed using SPSS 14 .

\section{Result:}

Out of total 1123 suspected TB cases, growth was detected in 461 cases. DST was performed in 397 specimens. But ultimately DST result was found in 363 cases. Remaining specimens were rejected due to contamination or some other different reasons. Out of 363 cases $79 \%$ were male and $21 \%$ were female. Age of all patients was 10 years and above.

Drug susceptibility testing for INH, RIF, EMB and SM was done. Resistance rates were found highest for INH. Among 363 cases, resistance rates for INH, RIF, EMB and SM were $76.03 \%, 71.63 \%, 27.55 \%$ and $55.65 \%$ respectively (Fig 1).

According to DST report, total 221 cases were detected as multi drug resistant TB (MDR-TB). Among them, $87 \%$ cases were MDR-TB in category II (CAT - II) failure and 13\% were MDR-TB in category I (CAT-I) failure and it was $0 \%$ in new cases (Fig.-2).

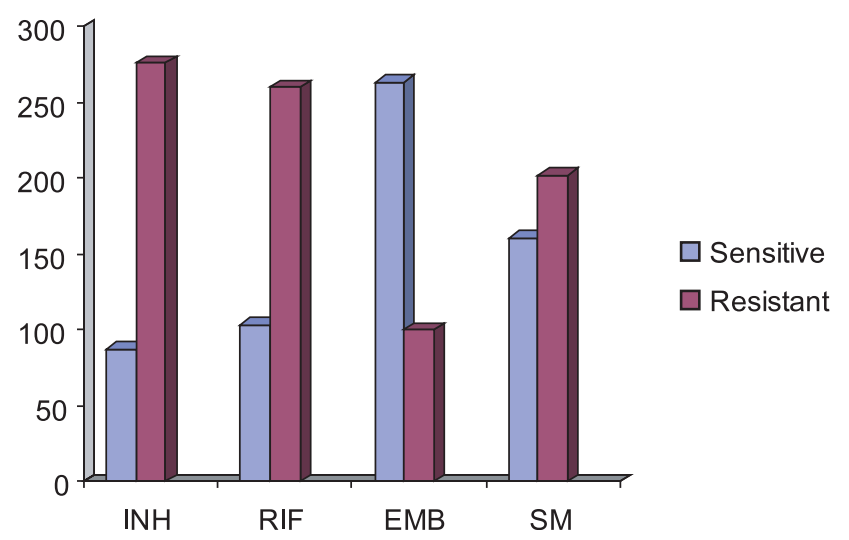

Fig 1: Drug susceptibility testing for different anti mycobacterial drugs.

MDR-TB

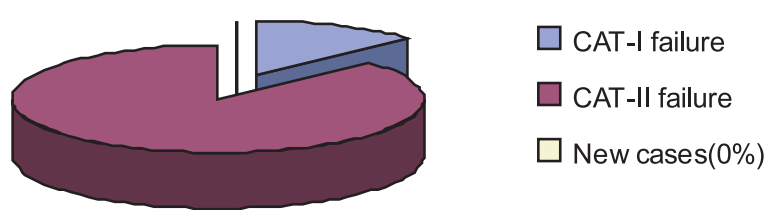

Fig.-2: Pattern of MDR-TB among different category of Tuberculosis patients.

\section{Discussion:}

To estimate the drug resistance of MTB, it is extremely important to evaluate the epidemiology and control of tuberculosis. Like many developing countries, in Bangladesh, drug resistant tuberculosis is increasing and is a significant threat to tuberculosis control because there are few drugs effective against TB. ${ }^{4}$ Sporadic survey by Damien Foundation and ICDDR,B shows overall prevalence of MDR $2-5.5 \%$. It was significantly higher among persons who previously had received tuberculosis treatment of one month or more; varies from $5.6 \%$ to $15.4 \% .{ }^{5}$ In this context, DOTS strategy seems to be an answer to control tuberculosis but rapid emergence of drug resistance may arise with a fixed regimen and not with a regimen based on drug susceptibility profile. This occurs because of lack of information about previous history of treatment.

In the present study, DST had been performed for INH, RIF, EMB and SM. The resistance rate was highest for INH (76.03\%) followed by RIF (71.63\%), EMB (27.55\%) and SM (55.65\%). In the present study INH and RIF, resistance was found in two third of the patients, which is very high while resistance to SM and EMB was slightly low. 
Culture and susceptibility testing has been done in the laboratory of NIDC\&H between January 2001 and August 2004 revealed completely different results. DST results showed among the failure cases 50\% were resistant to RIF, 96\% were resistant to INH, $77 \%$ were resistant to EMB and 19\% were resistant to EMB. ${ }^{1}$ Our study completely differs from a study done by B. Mahadev et. al. in India. The level of resistance to INH was $10.3 \%$ and to RIF was $2.5 \%$ in Hoogli district and $2.5 \%$ for INH and $0.7 \%$ for RIF in Mayurbhanj district. ${ }^{6}$ Another study performed by Iqbal $R$ et. al showed overall resistance to rifampicin was $26.6 \%$, to isoniazid $23.5 \%$, streptomycin $19.5 \%$, ethambutol $11.8 \%$, and to pyrazinamide $29.7 \%{ }^{7}$

In our study, according to DST report, total 221 cases were detected as MDR-TB. Among them, $87 \%$ cases were MDR-TB in category II (CAT - II) failure and $13 \%$ were MDR-TB in category I (CAT - I) failure and it was $0 \%$ in new cases. On a national scale survey in Bangladesh, the treatment success rate in new smearpositive cases registered for treatment in 2002 was 84\%. This indicates that MDR-TB should not be a problem in new

cases. However, treatment success reported among previously treated cases was much lower: $68.7 \%$ in relapses, $71.5 \%$ in failures of category 1 , and $69.1 \%$ in treatment after default. In total, in 2002, 325 patients failed on category 1 regimen and 82 failed on category 2 regimen. ${ }^{1}$ Data from nine sites in India show that drug resistance among new case is relatively low; new data from Gujrat indicate that, at 17.2\%, MDRTB among re-treatment cases is higher than previously anticipated. ${ }^{8}$

Limited studies to date suggest that the prevalence of MDR-TB is around 3\% among newly diagnosed cases and $15 \%$ among previously treated patients in Bangladesh. Drug- resistant survey (DRS) conducted Damien Foundation in 1995 and 2001 suggest a fall in the prevalence of MDR-TB from $0.7 \%$ to $0.4 \%$ among new and from $6.8 \%$ to $3.0 \%$ among previously treated patients. ${ }^{9}$ The National TB Control Programme (NTP) proposes to conduct a DST for all category II and I failures. ${ }^{8}$

The emergence of MDR-TB poses a significant threat to TB control activities through out the world. The complexity of MDR-TB management makes it essential to create new skills to design, plan, implement, and monitor interventions for the management of MDR-TB. More surveillance and immediate therapeutic interventions should be performed in order to combat the threat of MDR-TB to the general population.

\section{References:}

1. National Tuberculosis Programme, Bangladesh .Report of the Third Review - September 2004 .Available at:http://www.searo.who.int/LinkFiles/ Reports_sea-tb-273.pdf Accessed May 102009.

2. Zaman K, Hossain S, Yunus M, et. al. Tuberculosis in Bangladesh: A 40-year Review .ICDDR,B, National Tuberculosis Control Programme, Directorate General of Health Services, Bangladesh, Bangladesh Rural Advancement Committee, Bangladesh, and Johns Hopkins Bloomberg School of Public Health, USA. Available at: http:// www.icddrb.org/pub/publication.jsp? classificationID=1\&pubID=7981 Accessed May 10 2009.

3. World Health Organization. World health organization reports highest rates of drug-resistant tuberculosis to date. Available at: http:// www.who.int/tb/features_archive/drsreport_ launch_26feb08/en/ Tuberculosis (TB) Accessed May 102009.

4. Bengisun JS, Karnak D, Palabiyikoglu I, Saygun N. Mycobacterium tuberculosis drug resistance in Turkey, 1976-97. Scand J Infect Dis 2000; 32: 50710.

5. Salim MAH. New hope in the treatment of MDR TB. Damien Foundation Bangladesh. Available at:www.lungbd.org/pdf/21.pdf Accessed May 10 2009 .

6. Mahadev B, Kumar P, Agarwal SP, Chauhan LS, Srikantaramu N. Surveillance of drug resistance to anti-tuberculosis drugs in districts of hoogli in west bengal and mayurbhanj in orissa. Indian Journal of Tuberculosis 2004.

7. Iqbal R, Shabbir I, Khan SU, Saleem S, Munir K. Multidrug Resistance Tuberculosis in Lahore. Pak J Med Res 2008; 47: 1.

8. World Health Organization. Management of drugresistant tuberculosis. Report of workshop, Faridabad, Haryana, India, 6-10 August, 2007.

9. Mahmud AM, Rahman MM, Hasan MR, et al. Multi-drug resistant and extensively drug resistant TB and the DOTS-PLUS project in Bangladesh. Available at: www.lungbd.org/pdf/19.pdf Accessed May 102009. 